\title{
Tendon healing: an overview of physiology, biology, and pathology of tendon healing and systematic review of state of the art in tendon bioengineering
}

\author{
Sebastian A. Müller • Atanas Todorov • \\ Patricia E. Heisterbach • Ivan Martin • \\ Martin Majewski
}

Received: 1 April 2013/Accepted: 7 September 2013/Published online: 21 September 2013

(C) Springer-Verlag Berlin Heidelberg 2013

\begin{abstract}
Purpose Tendon injuries vary from acute rupture to chronic tendinopathy. For an optimal treatment of either condition, a profound knowledge is essential. Therefore, this article shall give an overview of physiology, biology, and pathology of tendon healing and state of the art in tendon bioengineering.

Methods For a preferably comprehensive survey, the current literature listed in PubMed and published in English peer-reviewed journals (March 2013) was systematically reviewed for tendon healing and tendon bioengineering including cytokine modulation, autologous sources of growth factors, biomaterials, gene therapy, and cell-based therapy. No differentiation was made between clinical and preclinical in vitro investigations.

Results Tendon healing happens in certain stadiums of inflammation, formation, and remodelling. An additional process of "collagen recycling" close to the healing site has been described recently. With increasing comprehension of physiology and pathology of tendon healing, several promising approaches in tendon bioengineering using growth factors, biomaterials, gene therapy, or cell-based therapy are described. However, only some of these are already used routinely in clinics.

Conclusion Strong and resistant tendons are crucial for a healthy musculoskeletal system. The new approaches in tendon bioengineering are promising to aid physiological
\end{abstract}

S. A. Müller $(\varangle) \cdot$ P. E. Heisterbach · M. Majewski Department of Orthopedic Surgery, University Hospital Basel, Spitalstrasse 21, 4031 Basel, Switzerland

e-mail: muellerse@uhbs.ch

A. Todorov - I. Martin

Department of Biomedicine, University Hospital Basel,

Hebelstrasse 20, 4031 Basel, Switzerland tendon healing and thus resulting in a stronger and more resistant tendon after injury. The growing knowledge in this field will need to be further taken into clinical studies so that especially those patients with prolonged courses, revision surgery, or chronic tendinopathy and high-demanding patients, i.e., professional athletes would benefit.

Level of evidence II.

Keywords Tendon - Tendon healing .

Bioengineering · Growth factor - Stem cell

\section{Introduction}

Tendons are the functional link between the dynamic and the static part of the musculoskeletal system transferring muscle contraction to the skeletal system and thus leading to motion. Consequently, function and motion are impaired in the case of tendon injury varying from acute traumatic ruptures to chronic overuse and degenerative tendinopathy. But even with improved therapeutic strategies (non-surgical, surgical, and rehabilitation techniques), outcomes remain inconsistently: Repaired tendon tissue rarely achieves functionality equal to that of the pre-injured state $[20,29]$. The final tensile strength of healed tendon may be reduced by up to $30 \%$ [40]. For optimal treatment, enhanced understanding of tendon physiology, degenerative pathology, and healing processes in ruptured tendons is necessary. Due to their similar structure, the healing of ligaments is usually compared and subsumed to tendon healing.

Among others, growth factors and cytokines modulate the differentiation of tendons during embryogenesis and also play an important role during the healing process of tendons [17]. 
Physiologically, growth factors are synthesized and secreted by a variety of inflammatory cells, platelets, fibroblasts, epithelial cells, and vascular endothelial cells. By binding to the external receptors, growth factors have the potential to directly influence cellular mitogenesis and thus the healing cascade [35]. In general, growth factors are effective in increasing the cellularity and the overall tissue volume at the repair site, resulting in increasing failure loads on biomechanical testing. However, these failure loads become less significant if normalized to the volume or cross-sectional area of the repaired tissue [40, 41]. This implies that growth factors are able to improve the strength of the repair by promoting the formation of more scar tissue (i.e. the structural properties are improved, but the material properties are not improved).

Further advances in bioengineering including the use of mesenchymal stem cells and biocompatible scaffolds are promising [20, 30, 32]. For a better understanding and treatment of tendon injury, this article shall first give an overview of tendon physiology, biology, and pathology of tendon healing before reviewing the state of the art in tendon bioengineering, possible being the future in treatment of tendon healing especially in prolonged cases.

\section{Materials and methods}

Based on the well-known principles of tendon healing, the current literature listed in PubMed (March 2013) was systematically reviewed for tendon healing with respect to bioengineering, or growth factor, or cytokine therapy, or gene therapy, or cell-based therapy, and thoroughly analysed. The search produced 657 results in PubMed. Duplicates were excluded and only Level 1 and Level 2 investigations published in English peer-review journals were respected for the systemic review part, leading to a total number of 45 studies (1 prospective randomized controlled study and 44 controlled laboratory studies: 10 controlled in vitro studies; 33 controlled animals studies; 1 combined in vitro/in vivo study). Finally, the topics and the results of the reviewed articles were subdivided into tendon healing with respect to: collagen recycling, mechanical load, cytokine modulation, autologous sources of growth factors, biomaterials, gene therapy, and cell-based therapy. Previously, the principals of tendon healing are described and listed by: structure and function of tendons, molecular base of tendon development and tendon neoformation, and biology and pathology of tendon healing.

The aim of this article was to give a possible broad overview of bioengineering in tendon healing and not to focus only on one single pathway. As a consequence, the main limitation of this work is the heterogeneity of different approaches in tendon healing and the limited number of articles on each topic. Thus, detailed comparison of different studies is not possible. Moreover, most of them are quite modern having only been investigated in in vitro or animal studies, but not yet routinely in clinics ( 1 clinical study vs. 44 controlled laboratory studies).

\section{Results}

Overview of biology and pathology of tendon healing

\section{Structure and function of tendons}

The high amount of collagen (70\% of tendon dry weight) and the parallel fibre orientation account for the high tensile strength of tendons, the highest in the body. Type I collagen is the predominate type of collagen (95\%), whereas collagen type III and V and glycosaminoglycan amount to the other $5 \%$. The collagen forms fibrils, which are organized into bundles together with nerves and blood vessels. The bundles are covered by epitenon and paratenon, sheets of connective tissue leading vessels and serving as a reserve for tenocytes. A small amount of synovial fluid between epitenon and paratenon allows smooth motion of the tendons.

\section{Molecular base of tendon development and tendon neoformation}

Embryogenic development of tendon tissue is highly associated to the development of the corresponding muscle [6]. The basic helix-loop-helix transcription factor scleraxis is a specific marker of tendon development, expressed in mature tendons and ligaments of the limbs and trunk, as well as in their progenitors.

The type II transmembrane glycoprotein tenomodulin is highly expressed in developing as well as in mature tendons. Along with scleraxis, tenomodulin is a candidate marker gene for tenocytes. Knockdown of tenomodulin reduces tenocyte proliferation [37]. However, molecular mechanisms in regulation of tendon development and neoformation are not yet totally understood and further scientific work is needed before therapeutic use is conceivably.

\section{Biology and pathology of tendon healing}

Tendon healing is organized into distinct stages [41]. After trauma, blood collects and clots at the site of injury, introducing the haemorrhagic stage of tendon healing. Typically, platelets degranulate and release a great amount of cytokines and growth factors. Next follows the stage of inflammatory tendon healing, where neutrophils and 
macrophages invade the haematoma and begin with the phagocytosis of necrotic material and pieces of extracellular matrix. This stage can last between 3 and 7 days after tendon injury. Extrinsic cells from the peritendinous soft tissue such as tendon sheath, fascia, periosteum, and subcutaneous tissue but also intrinsic cells from the epitenon and endotenon migrate and proliferate in the area of tendon injury. Together, they form the granulation tissue present in the so-called proliferative stage. This immature tissue synthesizes mainly collagen type III from the fifth day of tendon healing onward.

The initial collagen fibres are not yet oriented in parallel but they already contribute to the biomechanical strength. Up to the fifth week, the amount of collagen increases steadily and the repair callus reaches its largest size [35]. In the fourth week, intrinsic fibroblasts mainly from the endotenon start to proliferate increasingly. After about 40 days, these intrinsic fibroblasts play the most active role in tendon healing, resorbing collagen actively, and producing new collagen at the same time. The tendon tissue matures and the fibres are orientated more longitudinally according to the tension forces. This formative phase lasts for about 2 months.

Finally, the maximal biomechanical strength is accomplished in the remodelling phase, when the physiologic load is brought back to the tendon. The collagen fibres become more organized in the longitudinal axis and more cross-linked. Moreover, the type III collagen produced during the formative phase is replaced by the mechanically more resistant type I collagen. As the mechanical tissue properties improve, the callus transverse area gradually decreases. However, the tendon that has been remodelled over the following months remains hypercellular, the amount of type III collagen is higher with less potential for cross-linking the fibres as type I, and the collagen fibrils are thinner, leading to inferior biomechanical qualities compared to the uninjured tendon [35].

Systematic review of state of the art in tendon bioengineering

\section{Collagen recycling}

Apart from the classical stages of tendon healing by digestion and collagen de novo synthesis, recent investigations have suggested a kind of collagen recycling in the intrinsic tendon healing. Electron microscopy of corresponding cross-sectional areas of rat Achilles tendon 10 days after being cut and re-sutured showed smaller diameters of collagen bundles in the healing tendon compared to the uninjured side. There seems to be a parallel ongoing process of recycling collagen fibres in terms of increasing early tensile strength by reconnecting fibrils on either side of the repair site with fibroblast-controlled collagen fibril segments liberated from the adjacent tendon ends. The recycling process of collagen in tendon repair could cause a temporary weakness of the tendon as collagen from the adjacent areas is provided for the healing process. This could explain the post-operative rupture of flexor tendons next to the site of previous repair observed in hand surgery $[16,22]$.

\section{Influence of mechanical load on tendon properties}

Tenocytes in particular have the ability to respond to different mechanical stresses by regulating their expression of extracellular matrix components and matrix degradation enzymes. Biomechanical cues help to structure the maturing tendon both during development and tendon healing and also help to keep its homeostasis.

Mechanical load leads to an upregulation of type III collagen mRNA expression and increased growth factor concentrations inducing cell proliferation, differentiation, and matrix formation in tendon tissue [27, 42]. Thus, some mechanical stimulation during tendon healing is beneficial for the proper organization of collagen fibres and prevention of adhesion formation. However, mechanical under- or overstimulation can have an adverse effect causing lipid accumulation, mucoid formation, and tissue calcification, all of them being markers of tendinopathy increasing the risk of re-rupture or microruptures [53].

Insertional tendinopathy represents an interesting case of differential effect of mechanical stimulation. Usually, the superficial tendon layer is healthy whereas the deep layer is pathological. The superficial portion is consistently under greater strain than the deep layer because of the greater distance to the central axis of rotation of the joint. This misconfiguration plays a central role in the so-called stress-shielding model. A proposed therapy consists of an incision of the superficial muscle layer, thus distributing the load equally to the deep and superficial portion. In stress-shielded sheep patellar tendons, a significant reduction in the structural and material properties was found compared to mechanically loaded controls. Moreover, restressing caused recovery of tendons mechanical properties [38].

\section{Cytokine modulation of tendon healing}

In a rat supraspinatus tendon model, numerous growth factors increased markedly 1 week after injury and returned to pre-injury levels by 16 weeks [50]. Interaction of four of these growth factors was observed in a rat Achilles tendon healing model [18]. Figure 1 shows the presence of different growth factors according to the healing phases in tendon healing. The possible most 
important growth factors in tendon healing are described in more detail hereafter:

$b F G F$ Basic fibroblast growth factor (bFGF) has among others angiogenic and mitogenic effects on ligament fibroblasts and is known to be involved in general wound healing [8]. An up-regulated mRNA for bFGF was found in the tendon wound environment, in tenocytes, and in the tendon sheath of fibroblasts [11]. As bFGF peaks in the early healing course $[18,50]$, it might be a factor with potential to promote the healing process of tendons. There is already one study showing an acceleration of the initial tendon-to-bone remodelling of rotator cuff tendons in rats by local application and even a regeneration and remodelling of rotator cuff defects reconstructed with acellular dermal matrix grafts [21].

PDGF Platelet-derived growth factor (PDGF) was first isolated from platelets, but can be produced by many different cells, including smooth muscle cells. PDGF induces the chemotaxis of fibroblasts, their proliferation rate, and the overall collagen synthesis. Thus, a positive effect on healing of the medial femoro-tibial collateral ligament of rats has been observed [3]. The PDGF receptor beta was expressed by the cells at the remodelling site of patellar tendons up to 6 months after injury, suggesting long time dependency of growth factors in tendon healing [9]. In an in vitro model of rat fibroblast, a PDGF stimulus led to an increased collagen synthesis by $300 \%$ [45].

BMP Bone morphogenetic proteins (BMP) stimulate bone formation but also tendon cell mitogenesis and tendon healing. During tendon embryogenesis, especially BMP12 and 13 cause the expression of elastin and collagen I. In healthy human Achilles tendons, BMP12, BMP13, and BMP14 are present. BMP2, BMP7, and BMP12 are involved in tendon-to-bone healing, resulting in an improved load to failure in a sheep infraspinatus model [25]. A BMP12 gene transfer delivered by a genetically modified muscle flap around the repaired site of rat Achilles tendons significantly improved the maximal failure load, the tendon stiffness, and the size of the rupture callus. Histologically, collagen fibres were more organized and parallel. The diameter of single fibrils and collagen fibres was increased compared to control. An earlier shift from fibroblasts to fibrocytes with less fat cells in the healing area was achieved in the BMP12 gene transfer group [28].

CDMP Cartilage-derived morphogenetic proteins (CDMP) belong to the BMP family and are involved in embryonic skeletal development, joint morphogenesis, and tendons formation [14]. In a rat model, CDMP-treated repaired supraspinatus tendons were significantly stronger than untreated tendons and histologic analysis showed more organized healing [33]. By CDMP injection, the mechanical strength of healing Achilles tendons in rabbits and the size of callus was increased significantly [15].

TGF $\beta$ The transforming growth factor $\beta$ family (TGF $\beta$ ) is a weak stimulator of tendon cell migration and mitogenesis, but can stimulate expression of robust extracellular matrix. All 3 TGF $\beta$ isoforms significantly increased collagen I an III production in cultured tendon fibroblasts [24]. Moreover, the production of other matrix proteins including fibronectins and glycosaminoglycans is stimulated by TGF $\beta$. TGF $\beta$ is known to promote the maturation of monocytes into macrophages, which amplify the inflammatory response and remodel the wound site after neutrophil infiltration. Elevated levels of TGF $\beta$ were also found in rabbit flexor tendons after injury [10]. A single dose of TGF $\beta 1$ antibody during rabbit flexor tendon repair resulted

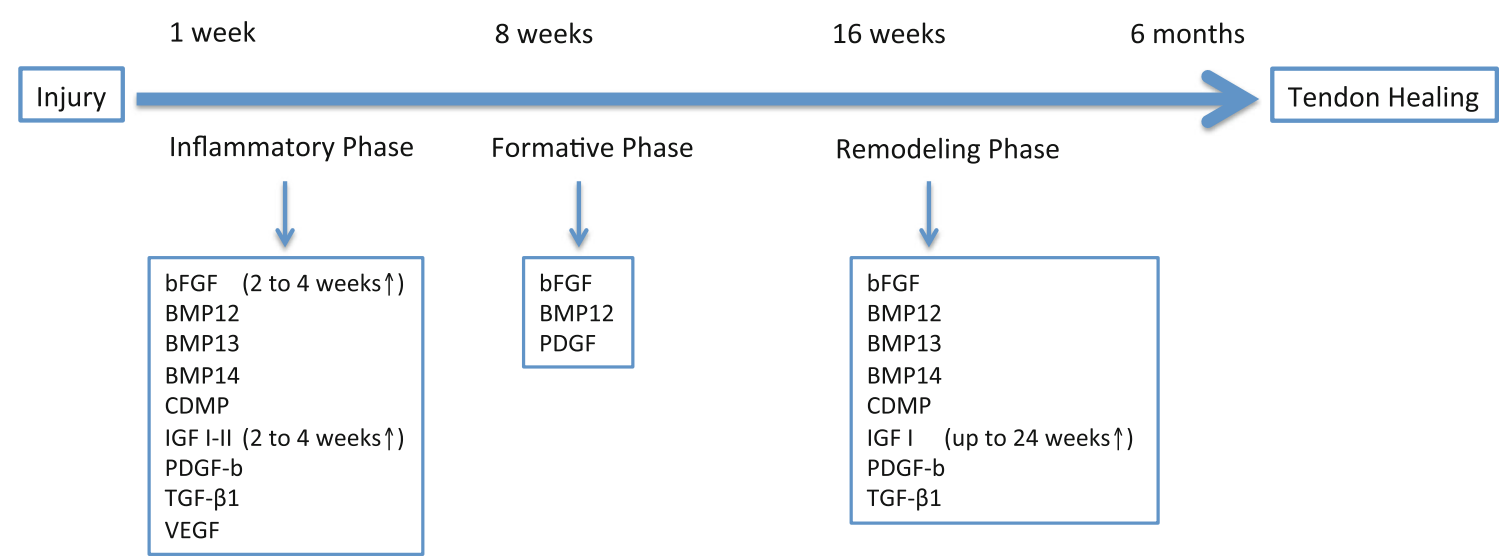

Fig. 1 As the healing process passes through the 3 phases of inflammation, formation, and remodelling, several growth factors are expressed. Modified from [35] 
in less scarring with an increased range of motion after surgery compared to the control group [12].

IGF Insulin-like growth factor (IGF) has a stimulatory effect on many cell types, including cartilage, bone, muscle, and tendon cells [43] and has been investigated in several animal studies: IGF induces tenocyte migration, division, matrix expression, and collagen synthesis. The latter seems to be site dependent producing more collagen in rabbit flexor tendons compared to Achilles tendons [1, 44].

VEGF Vascular endothelial growth factor (VEGF) is an endothelial mitogen that promotes angiogenesis and increases capillary permeability. The expression of VEGF mRNA peaked 10 days after surgery of canine flexor tendons [4]. A significant increase in tensile strength 2 weeks but not 4 weeks after surgical repair and treatment with VEGF of transected rat Achilles tendons has been observed [52].

\section{Autologous sources for a variety of growth factors}

Platelet-rich plasma (PRP) is an autologous blood product consisting of a limited volume of plasma containing an enriched concentration of platelets. Both plasma and platelets are rich sources of bioactive proteins, including growth factors, extracellular matrix proteins and other signalling molecules. The observed effects of PRP are attributed to the delivery of growth factors and proinflammatory mediators to damaged tissue. The delivery of these factors is especially valuable in situations of low blood flow or hypocellularity, two known features of ligaments and tendons. Hence, it does not come as a surprise that these tissues may substantially benefit from the application of PRP. Platelets are the most prevalent cell type in PRP and contain within their alpha granules and dense granules high quantities of growth factors and cytokines. Among others, the alpha granules contain PDGF, TGF $\beta$, IGF1, VEGF, and epidermal growth factor (EGF), whereas the dense granules contain neuromodulators and inflammatory modulators such as histamine and serotonin. By exposure to collagen, thrombin, or calcium platelets release these growth factors and cytokines [13].

Consequently, PRP should be an excellent supplier of the above-mentioned growth factors. However, the treatment of chronic Achilles tendinopathy in a randomized, double blind, placebo-controlled trial demonstrated no significant differences between patients managed with either PRP or saline solution injections [47]. On the other hand, repaired superficial digital flexor tendons in front limbs of horses had a higher failure strength and greater elastic modulus 24 weeks post-operatively after being treated with PRP compared to the control group with only saline injection. Histologically, the PRP group demonstrated better collagen organization and increased metabolic activity [5]. One reason for the above-mentioned inconsistent clinical findings could be the not yet very clear dose-response relationship of PRP. A high amount of PRP should support wound healing yet some authors think that overdosing of PRP leads to a poorly differentiated scar tissue by overstimulated cells. Some of the released growth factors might even have negative effects causing increased inflammation or suppression of osteoclasts [39].

Recently, bio-enhanced anterior cruciate ligament (ACL) repair by suturing bioactive materials in between the torn ACL-ends has been investigated. The idea is that bioactive material cannot be washed out by the circulating synovial fluid but can release growth factors to enhance cellular activity. A porcine model with a collagen-platelet composite inserted into the ACL rupture site showed defect filling by fibroblast as well as a return to physiological levels of mechanical strength. Comparing primary ACL suture repair to collagen-platelet augmented repair showed significant increases in stiffness, load at yield and maximal tensile load up to 14 weeks post-operatively [23].

Another natural source for a variety of growth factors including anti-inflammatory cytokines is autologous conditioned serum (ACS). Among the anti-inflammatory cytokines are interleukins (IL-4, IL-10, IL-13) and IL-1 receptor antagonist. bFGF, HGF, and TGF $\beta$ are found as growth factors. In a rat model, Majewski could show a positive effect of ACS in Achilles tendon healing with accelerated organization of repair tissue, an increase in collagen mRNA, an increased collagen deposition reflected by tendon thickness and an increased mechanical strength, all indicators for an improved healing with a lower risk of re-rupture. Nevertheless, the repaired tendons remained biomechanically inferior to native control tendons [30].

\section{Biomaterials in tendon healing}

Especially, ACL and rotator cuff injuries are associated with a defunct or missing tendon part. Though auto- and allografts can be used, availability is restricted and complications may arise due to the donor site morbidity or, in case of allografts, unforeseen transmission of infection.

Using a collagen-platelet composite, knee laxity after $\mathrm{ACL}$ reconstruction could be reduced in gout and biomechanical and histological healing could be enhanced [23]. Recently, a promising attempt using a nanofibre scaffold loaded with mesenchymal stem cells and growth factors in an animal model has been described showing an increase in DNA in the treated group [32]. 
However, only very little is understood about the effectiveness of growth factor or stem cell-loaded scaffolds in tendon healing so far.

\section{Gene therapy}

A different strategy for a longer and constant growth factor release in the healing site is the transfer of genetic material (DNA) to cells using viral or non-viral vectors or direct gene transfer. The cells incorporate the genetic material and produce the desired growth factors over a substantial period of time.

There are some studies in the literature investigating gene therapy in transected tendon models, of which the results may also be applied in tendinopathy. Application of BMP12 by transfected tenocytes at the laceration site led to a twofold increase in tensile strength and improved load to failure of the tendons after 4 weeks [26]. In another rat model, tenocytes were transfected by a VEGF or a PDGF carrying plasmid. PDGF gene transfer led to a significant increase in type I collagen and PDGF gene expression, whereas the VEGF gene transfer only increased expression of the TGF $\beta$ gene but not significantly the expression of the collagen type I and III genes [48]. As already mentioned above, Majewski has shown that the implantation of transfected muscle flaps expressing BMP12 or TGF $\beta$ is beneficial for tendon healing [28, 31].

\section{Cell-based therapy}

Combining the above-mentioned methods such as the use of growth factors, biomaterials and even transfected cells, it is the goal of cell-based therapy to provide functional grafts that satisfy several requirements: The graft should have good mechanical properties, it should begin remodelling and integration into host tissue immediately and it should stimulate, improve and accelerate the repair process. Clearly, cells provided in the graft should be responsible for stimulation of repair, maintenance of homeostasis and reduction in inflammation rather than addition of huge cell numbers.

Autologous cell sources including adipose tissue, muscle, synovia, periosteum, tendon-derived stem cells, dermal fibroblasts, and stem cells derived from umbilical cord or peripheral blood have been evaluated, next to the commonly used bone marrow-derived mesenchymal stem cells (MSC).

Expanded adipose-derived stem cells have been shown to improve healing in flexor tendons [46]. A subset of these cells, the vascular stromal cell fraction, has been shown to improve histological characteristics and reduce the inflammation in an equine collagenase-induced tendonitis model [34].
In adults, MSC are mainly found in bone marrow, but also in muscle, fat, skin, and around blood vessels. MSC have the potential to differentiate into a variety of specialized mesenchymal tissues such as bone, cartilage, muscle, tendon, ligament, fat, and marrow stroma. For example, muscle-derived progenitor cells differentiated into tenoblasts and were further incorporated into collagen bundles in supraspinatus tendons of nude rats [36]. Structural and material properties in a rat Achilles tendon defect model of $1 \mathrm{~cm}$ were twofold better using a MSC-collagen gel composite compared to the suture alone. The MSC group was larger in cross section and histologically better organized [51]. Comparable results were found in rabbit Achilles tendons, rabbit patellar tendons, and hen flexor tendons $[2,7,19]$.

More recently, also foetal-derived embryonic stem cells (ESC) have been used for tendon repair in an equine model of enzymatic or physical tendonitis, showing a highly improved healing with no activation of the immune system even though allogenic transplantation was performed [49]. Though results may be promising, use of these cells is ethically problematic and there is still some debate ongoing. Better alternatives are the recently developed induced pluripotent cells (iPSC), which can be produced through transfection of adult differentiated cells with Oct4, Sox2, Klf4 and c-Myc and display pluripotent markers and behaviour. As ESC, iPSC can be derived allogenically and stored for usage, yet they are not as ethically problematic.

\section{Discussion}

Not only due to its bradytroph nature, tendon healing is a complex process. Results of tendon treatment in daily practice are inconsistent. There is a lot of new knowledge about the healing mechanisms but only very little has been established in clinical practice as far as the current investigations on tendon tissue engineering are mainly preclinical studies. The aim in treatment of acute or chronic tendon pathologies must be, to come as close as possible to a normal tendon with comparable properties. A great challenge lies in degenerated tissues. For optimized and rapid tendon healing, a high expression of type I collagen is extremely important. The conversion of initially produced type III collagen into type I should be induced preferably early in tendon healing. Additionally, a certain amount of mechanical load is crucial for tendon healing as mentioned. However, the right amount and timing of mechanical load have not yet been defined, knowing that mechanical overand under-stimulation can have adverse effects. An early passive or active-assistive mobilization after surgical reconstruction seems to be reasonably. No one would let a 
patient with a reconstructed tendon neither maximally strain nor totally immobilize the corresponding joints. But hart data in the literature is missing, particularly as this could vary from each body region or even the tendon itself. Appropriate clinical studies would be desirably.

No doubt, growth factors play an important role in the healing course of tendon tissue. The clinician could be inclined to try to improve tendon healing by external application of one or several growth factors. The question is how and in which concentration to administer them to the healing site and which one of them would be the best to use. A direct local injection would have the benefit of being simple and quick. However, there is an overflow loss in local injection and only a short duration of the actual stimulation due to the short half-life of growth factors. This is in direct contrast to the fact that tendon healing takes months to years to complete, even though several animal studies have shown the effectiveness of local injections of growth factors. A repetitive or continuous administration could be an alternative. However, the attempts of a continuous external application in animal models have failed so far due to technical problems. Moreover, results with an unspecific "cocktail" of growth factors using PRP or ACS have been inconsistent as well. Perhaps, the best administered concentration of PRP or ACS is not known. Moreover, it remains unclear which one or which ones of the growth factors would be responsible for a given effect.

An even more appealing idea is a functional biomaterial that actively supports cell adhesion, migration, and proliferation and accelerates tendon healing by a targeted delivery of growth factors and other stimulatory molecules. This model could control the localization and release kinetic of any given stimulant. The idea is to reduce the overflow loss and to have a longer duration of the effect. Moreover, scaffolds can function as a guiding structure for migrating cells and the healing tissue.

Gene therapy also holds great promise, yet it is still a long way from a complete therapy. Cell-based therapy, especially in combination with biomaterials, growth factors, and gene therapy, seems to be the most thorough and promising approach but research and development in this direction has barely just begun and has still some way to go. Figure 2 shows the current investigated fields of tendon bioengineering, possibly two or more of them could be combined to increase the effect.

Based on the numerous preclinical investigations, the next conceivable step could be to establish one realistic model using for example some of the known growth factors combined with a biocompatible scaffold. Such a model could be implemented in the near future and has the possible potential to bridge tendon defects known from rotator cuff tears or Achilles tendon revisions, for instant. To the authors, especially bFGF, BMP12, and TGF $\beta$ seem to have

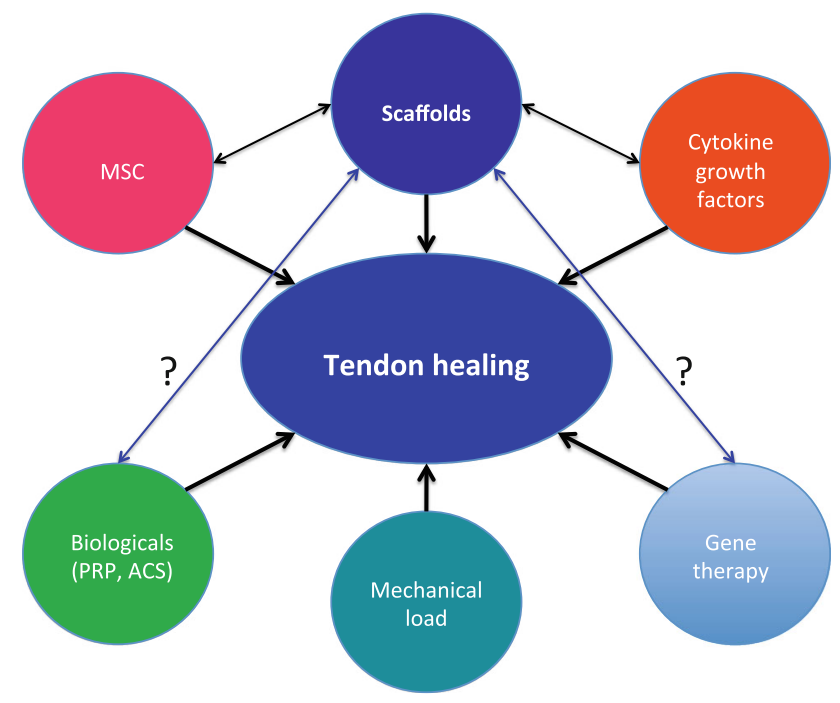

Fig. 2 Tendon healing is influenced by several factors, which can be used in tissue engineering of healing tendon. Each factor is known to have its impact. Possible the impact can be optimized by combination of factors or loading cytokine growth factors to scaffolds

the power to influence the regeneration progresses in tendon healing and increase the amount of collagen type I. The growth factors could be loaded on a collagen scaffold for a continuous and prolonged release but also as an anatomic landmark for the ingrowth of healing cells. Having a successful model, the next step would be to implement this model into a clinical study.

\section{Conclusion}

Several different promising approaches in tissue engineering of tendon healing are of current interest. Some improvements have been achieved and are implemented in clinical practice using biological "cocktails with growth factors" (PRP and ACS). Using specific agents either recombinant growth factors or scaffolds or cell-based strategies are promising even if some of these currently seem to be far away of potential clinical use.

\section{References}

1. Abrahamsson SO, Lohmander S (1996) Differential effects of insulin-like growth factor-I on matrix and DNA synthesis in various regions and types of rabbit tendons. J Orthop Res 14:370-376

2. Awad HA, Butler DL, Boivin GP, Smith FN, Malaviya P, Huibregtse B, Caplan AI (1999) Autologous mesenchymal stem cell-mediated repair of tendon. Tissue Eng 5:267-277

3. Batten ML, Hansen JC, Dahners LE (1996) Influence of dosage and timing of application of platelet-derived growth factor on early healing of the rat medial collateral ligament. J Orthop Res 14:736-741 
4. Bidder M, Towler DA, Gelberman RH, Boyer MI (2000) Expression of mRNA for vascular endothelial growth factor at the repair site of healing canine flexor tendon. J Orthop Res 18:247-252

5. Bosch G, van Schie HT, de Groot MW, Cadby JA, van de Lest CH, Barneveld A, van Weeren PR (2010) Effects of platelet-rich plasma on the quality of repair of mechanically induced core lesions in equine superficial digital flexor tendons: a placebocontrolled experimental study. J Orthop Res 28:211-217

6. Brent AE, Schweitzer R, Tabin CJ (2003) A somitic compartment of tendon progenitors. Cell 113:235-248

7. Cao Y, Liu Y, Liu W, Shan Q, Buonocore SD, Cui L (2002) Bridging tendon defects using autologous tenocyte engineered tendon in a hen model. Plast Reconstr Surg 110:1280-1289

8. Chan BP, Chan KM, Maffulli N, Webb S, Lee KK (1997) Effect of basic fibroblast growth factor. An in vitro study of tendon healing. Clin Orthop Relat Res 342:239-247

9. Chan BP, Fu SC, Qin L, Rolf C, Chan KM (2006) Supplementation-time dependence of growth factors in promoting tendon healing. Clin Orthop Relat Res 448:240-247

10. Chang J, Most D, Stelnicki E, Siebert JW, Longaker MT, Hui K, Lineaweaver WC (1997) Gene expression of transforming growth factor beta-1 in rabbit zone II flexor tendon wound healing: evidence for dual mechanisms of repair. Plast Reconstr Surg 100:937-944

11. Chang J, Most D, Thunder R, Mehrara B, Longaker MT, Lineaweaver WC (1998) Molecular studies in flexor tendon wound healing: the role of basic fibroblast growth factor gene expression. J Hand Surg Am 23:1052-1058

12. Chang J, Thunder R, Most D, Longaker MT, Lineaweaver WC (2000) Studies in flexor tendon wound healing: neutralizing antibody to TGF-beta1 increases postoperative range of motion. Plast Reconstr Surg 105:148-155

13. Dohan Ehrenfest DM, Rasmusson L, Albrektsson T (2009) Classification of platelet concentrates: from pure platelet-rich plasma (P-PRP) to leucocyte- and platelet-rich fibrin (L-PRF). Trends Biotechnol 27:158-167

14. Erlacher L, McCartney J, Piek E, ten Dijke P, Yanagishita M, Oppermann H, Luyten FP (1998) Cartilage-derived morphogenetic proteins and osteogenic protein-1 differentially regulate osteogenesis. J Bone Miner Res 13:383-392

15. Forslund C, Aspenberg P (2003) Improved healing of transected rabbit Achilles tendon after a single injection of cartilage-derived morphogenetic protein-2. Am J Sports Med 31:555-559

16. Goldfarb CA, Harwood F, Silva MJ, Gelberman RH, Amiel D, Boyer MI (2001) The effect of variations in applied rehabilitation force on collagen concentration and maturation at the intrasynovial flexor tendon repair site. J Hand Surg Am 26:841-846

17. Grotendorst GR (1988) Growth factors as regulators of wound repair. Int J Tissue React 10:337-344

18. Heisterbach PE, Todorov A, Flückiger R, Evans $\mathrm{CH}$, Majewski M (2012) Effect of BMP-12, TGF- $\beta 1$ and autologous conditioned serum on growth factor expression in Achilles tendon healing. Knee Surg Sports Traumatol Arthrosc 20:1907-1914

19. He Q, Li Q, Chen B, Wang Z (2002) Repair of flexor tendon defects of rabbit with tissue engineering method. Chin J Traumatol 5:200-208

20. Hogan MV, Bagayoko N, James R, Starnes T, Katz A, Chhabra AB (2011) Tissue engineering solutions for tendon repair. J Am Acad Orthop Surg 19:134-142

21. Ide J, Kikukawa K, Hirose J, Iyama K, Sakamoto H, Mizuta $H$ (2009) The effects of fibroblast growth factor-2 on rotator cuff reconstruction with acellular dermal matrix grafts. Arthroscopy 25:608-616

22. Ingraham JM, Weber RA, Childs EW (2011) Intrinsic tendon healing requires the recycling of tendon collagen fibril segments. J Hand Surg Eur 36:154-155
23. Joshi SM, Mastrangelo AN, Magarian EM, Fleming BC, Murray MM (2009) Collagen-platelet composite enhances biomechanical and histologic healing of the porcine anterior cruciate ligament. Am J Sports Med 37:2401-2410

24. Klein MB, Yalamanchi N, Pham H, Longaker MT, Chang J (2002) Flexor tendon healing in vitro: effects of TGF-beta on tendon cell collagen production. J Hand Surg Am 27:615-620

25. Kovacevic D, Rodeo SA (2008) Biological augmentation of rotator cuff tendon repair. Clin Orthop Relat Res 466:622-633

26. Lou J, Tu Y, Burns M, Silva MJ, Manske P (2001) BMP-12 gene transfer augmentation of lacerated tendon repair. J Orthop Res 19:1199-1202

27. Maeda E, Shelton JC, Bader DL, Lee DA (2009) Differential regulation of gene expression in isolated tendon fascicles exposed to cyclic tensile strain in vitro. J Appl Physiol 106:506-512

28. Majewski M, Betz O, Ochsner PE, Liu F, Porter RM, Evans CH (2008) Ex vivo adenoviral transfer of bone morphogenetic protein 12 (BMP-12) cDNA improves Achilles tendon healing in a rat model. Gene Ther 15:1139-1146

29. Majewski M, Schaeren S, Kohlhaas U, Ochsner PE (2008) Postoperative rehabilitation after percutaneous Achilles tendon repair: early functional therapy versus cast immobilization. Disabil Rehabil 30:1726-1732

30. Majewski M, Ochsner PE, Liu F, Flückiger R, Evans CH (2009) Accelerated healing of the rat Achilles tendon in response to autologous conditioned serum. Am J Sports Med 37:2117-2125

31. Majewski M, Porter RM, Betz OB, Betz VM, Clahsen H, Flückiger R, Evans CH (2012) Improvement of tendon repair using muscle grafts transduced with TGF- $\beta 1$ cDNA. Eur Cell Mater 23:94-101 Discussion 101-102

32. Manning CN, Schwartz AG, Liu W, Xie J, Havlioglu N, Sakiyama-Elbert SE, Silva MJ, Xia Y, Gelberman RH, Thomopoulos S (2013) Controlled delivery of mesenchymal stem cells and growth factors using a nanofiber scaffold for tendon repair. Acta Biomater 9:6905-6914

33. Murray DH, Kubiak EN, Jazrawi LM, Araghi A, Kummer F, Loebenberg MI, Zuckerman JD (2007) The effect of cartilagederived morphogenetic protein 2 on initial healing of a rotator cuff defect in a rat model. J Shoulder Elbow Surg 16:251-254

34. Nixon AJ, Dahlgren LA, Haupt JL, Yeager AE, Ward DL (2008) Effect of adipose-derived nucleated cell fractions on tendon repair in horses with collagenase-induced tendinitis. Am J Vet Res 69:928-937

35. Oliva F, Via AG, Maffulli N (2011) Role of growth factors in rotator cuff healing. Sports Med Arthrosc 19:218-226

36. Pelinkovic D, Lee JY, Engelhardt M, Rodosky M, Cummins J, Fu FH, Huard J (2003) Muscle cell-mediated gene delivery to the rotator cuff. Tissue Eng 9:143-151

37. Qi J, Dmochowski JM, Banes AN, Tsuzaki M, Bynum D, Patterson M, Creighton A, Gomez S, Tech K, Cederlund A, Banes AJ (2012) Differential expression and cellular localization of novel isoforms of the tendon biomarker tenomodulin. J Appl Physiol 113:861-871

38. Rumian AP, Draper ER, Wallace AL, Goodship AE (2009) The influence of the mechanical environment on remodelling of the patellar tendon. J Bone Joint Surg Br 91:557-564

39. Serhan CN, Savill J (2005) Resolution of inflammation: the beginning programs the end. Nat Immunol 6:1191-1197

40. Sharma P, Maffulli N (2005) The future: rehabilitation, gene therapy, optimization of healing. Foot Ankle Clin 10:383-397

41. Sharma P, Maffulli N (2006) Biology of tendon injury: healing, modeling and remodeling. $\mathbf{J}$ Musculoskelet Neuronal Interact 6:181-190

42. Skutek M, van Griensven M, Zeichen J, Brauer N, Bosch U (2001) Cyclic mechanical stretching modulates secretion pattern 
of growth factors in human tendon fibroblasts. Eur J Appl Physiol $86: 48-52$

43. Trippel SB, Wroblewski J, Makower AM, Whelan MC, Schoenfeld D, Doctrow SR (1993) Regulation of growth-plate chondrocytes by insulin-like growth-factor I and basic fibroblast growth factor. J Bone Joint Surg Am 75:177-189

44. Tsuzaki M, Brigman BE, Yamamoto J, Lawrence WT, Simmons JG, Mohapatra NK, Lund PK, Van Wyk J, Hannafin JA, Bhargava MM, Banes AJ (2000) Insulin-like growth factor-I is expressed by avian flexor tendon cells. J Orthop Res 18:546-556

45. Uggen JC, Dines J, Uggen CW, Mason JS, Razzano P, Dines D, Grande DA (2005) Tendon gene therapy modulates the local repair environment in the shoulder. J Am Osteopath Assoc 105:20-21

46. Uysal CA, Tobita M, Hyakusoku H, Mizuno H (2012) Adiposederived stem cells enhance primary tendon repair: biomechanical and immunohistochemical evaluation. J Plast Reconstr Aesthet Surg 65:1712-1719

47. de Vos RJ, Weir A, van Schie HT, Bierma-Zeinstra SM, Verhaar JA, Weinans H, Tol JL (2010) Platelet-rich plasma injection for chronic Achilles tendinopathy: a randomized controlled trial. JAMA 303:144-149
48. Wang XT, Liu PY, Tang JB (2005) Tendon healing in vitro: modification of tenocytes with exogenous vascular endothelial growth factor gene increases expression of transforming growth factor beta but minimally affects expression of collagen genes. J Hand Surg Am 30:222-229

49. Watts AE, Yeager AE, Kopyov OV, Nixon AJ (2011) Fetal derived embryonic-like stem cells improve healing in a large animal flexor tendonitis model. Stem Cell Res Ther 2:4

50. Würgler-Hauri CC, Dourte LM, Baradet TC, Williams GR, Soslowsky LJ (2007) Temporal expression of 8 growth factors in tendon-to-bone healing in a rat supraspinatus model. J Shoulder Elbow Surg 16:S198-S203

51. Young RG, Butler DL, Weber W, Caplan AI, Gordon SL, Fink DJ (1998) Use of mesenchymal stem cells in a collagen matrix for Achilles tendon repair. J Orthop Res 16:406-413

52. Zhang F, Liu H, Stile F, Lei MP, Pang Y, Oswald TM, Beck J, Dorsett-Martin W, Lineaweaver WC (2003) Effect of vascular endothelial growth factor on rat Achilles tendon healing. Plast Reconstr Surg 112:1613-1619

53. Zhang J, Wang JH (2010) Mechanobiological response of tendon stem cells: implications of tendon homeostasis and pathogenesis of tendinopathy. J Orthop Res 28:639-643 